\begin{tabular}{|c|c|}
\hline Title & A robust optimization based on adjoint variable method \\
\hline Author(s) & Maruy ama, T akay uki; W atanabe, Kota; Igarashi, Hajime \\
\hline Citation & $\begin{array}{l}\text { COMPEL : The International Journal for Computation and Mathematics in Electrical and Electronic Engineering, 29(6), } \\
\text { 1524 1532 } \\
\text { https://doi.org/10.1108/03321641011078599 }\end{array}$ \\
\hline Issue Date & 2010 \\
\hline Doc URL & http:/hdl.handle.net/2115/44988 \\
\hline Type & article (author version) \\
\hline File Information & COMPEL29-6_1524 1532.pdf \\
\hline
\end{tabular}

Instructions for use 


\section{A Robust Optimization Based on Adjoint Variable Method}

\section{ABSTRACT}

\section{Purpose}

This paper presents a new method to obtain robust solutions to electromagnetic optimization problems, solved with evolutional algorithms, which are insensitive to changes in design parameters such as spatial size, positioning and material constant.

\section{Design/methodology/approach}

To the adjoint variable (AVM) method is employed to evaluate the sensitivity of individuals in evolutional processes.

\section{Findings}

It is shown in the numerical examples where the present method is applied to optimization of a superconducting energy storage system and C-shape magnet that robust solutions are actually obtained which are insensitive to deviations in spatial sizes.

\section{Originality/value}

Unlike usual optimization methods, the present method takes deviation in the design parameters due to production errors and long-term changes into account. Moreover, the present method is limited to about twice the computational cost of non-robust optimization methods.

Keywords: adjoint variable method, optimization, finite element method, magnetostatic field, immune algorithm, sensitivity analysis

Type of paper: research paper

\section{INTRODUCTION}

Design optimization based on computational electromagnetism plays a crucial role in various electrical and electronic industries. In these optimization processes, it is usually assumed that the design parameters have no deviations or uncertainties which are ascribed to production errors, long-term changes in material properties and so on. To increase reliability and safety of products, it is important to take those effects into account in the design process. For this reason, the robust optimization methods which can treat deviations in the design parameters have been studied.

A robust optimization method has been presented in which the objective function values are sampled at grid points near to the candidate solution in the parameter space to evaluate its sensitivity [Steiner et al., 2004]. Although this method would provide reliable results, it requires great computational costs which grow in proportion to $2^{n}$ where $n$ denotes the number of design parameters. A robust optimization based on the genetic algorithm has also been presented [Maruyama and Igarashi, 2008], where random noises are added to design parameters to take the effect of the uncertainties into account. It is shown that this method can find robust optimal solutions which are insensitive to the parameter variations, and require no additional computational cost. However, it is difficult to impose the maximum acceptable deviations on the objective functions and constraints in this method.

In this paper, we apply the adjoint variable method (AVM) to the robust optimization to evaluate deviations in the objective function and constraint. Since the computational cost of AVM is independent of the number of design parameters, it would be suitable for the robust optimization. In computational electromagnetism, the AVM has been used in sensitivity analysis [e.g., Akel and Webb, 2006], topology optimization [Okamoto et al., 2006] and inverse analysis [e.g., Sikora, 1996]. However, the AVM has not been applied to the robust optimization. 
Let us consider a linear magnetostatic system for simplicity. The finite element (FE) equation is expressed in a matrix form as

$$
[K] \boldsymbol{a}=\boldsymbol{b},
$$

where $[K]$ is a symmetric sparse matrix whose entities are independent of $\boldsymbol{a}$. We now consider a function $f$ relevant to the performance or constraint of a magnetostatics system, which depends on design parameters $p_{i}$ (for $i=1,2, \ldots, m$ ). The purpose of sensitivity analysis is to compute the deviation of $f$ due to the parameter changes. The adjoint equation for (1) is defined by

$$
[K] \overline{\boldsymbol{a}}=\frac{\partial f}{\partial \boldsymbol{a}},
$$

where $\overline{\boldsymbol{a}}$ denotes the adjoint variable. By differentiating both sides of (1) with respect to $p$, which is one of the design parameters, we obtain

$$
[K] \frac{\partial \boldsymbol{a}}{\partial p}=-\frac{\partial[K]}{\partial p} \boldsymbol{a}+\frac{\partial \boldsymbol{b}}{\partial p} .
$$

By multiplying the adjoint vector $\overline{\boldsymbol{a}}$ from the left of (3), and taking its transpose, we have

$$
\frac{\partial f}{\partial p}=-\boldsymbol{a}^{t} \frac{\partial[K]}{\partial p} \overline{\boldsymbol{a}}+\frac{\partial \boldsymbol{b}^{t}}{\partial p} \overline{\boldsymbol{a}} .
$$

It is concluded that the sensitivity $\partial f / \partial p$ can be computed from (4) once we solve (1) and (2).

The standard deviation $\sigma_{f}$ in $f$ can be obtained from the sensitivities as follows:

$$
\sigma_{f}^{2}=\sum_{i}^{m} \sum_{j}^{m}\left(\frac{\partial f}{\partial p_{i}}\right)\left(\frac{\partial f}{\partial p_{j}}\right) \operatorname{Cov}\left(p_{i}, p_{j}\right) .
$$

When the design parameters are linearly independent for each other, (5) reduce to

$$
\sigma_{f}^{2}=\sum_{i}^{m}\left(\frac{\partial f}{\partial p_{i}}\right)^{2} \sigma_{p_{i}}{ }^{2}
$$

We next consider a non-linear magnetostatic system where magnetic saturation is taken into account. Because the entities in $[K]$ now depend on $\boldsymbol{B}$ in this situation, the derivative $\partial[K] / \partial p$ is written by

$$
\frac{\partial[K]}{\partial p}=\left(\frac{\partial[K]}{\partial p}\right)_{\boldsymbol{a}}+\frac{\partial[K]}{\partial \boldsymbol{a}} \frac{\partial \boldsymbol{a}}{\partial p},
$$

where the first term in right-hand-side of (7) denotes derivative of $[K]$ in terms of $p$ keeping $\boldsymbol{a}$ unchanged. Then the non-linear version of (3) is given by

$$
\left([K]+\left[K^{\prime}\right]\right) \frac{\partial \boldsymbol{a}}{\partial p}=-\frac{\partial[K]}{\partial p} \boldsymbol{a}+\frac{\partial \boldsymbol{b}}{\partial p},
$$

where the entities in $\left[K^{\prime}\right]$ which expresses the non-linear effect are given by

$$
K_{i j}^{\prime}=\sum_{k} a_{k} \frac{\partial K_{i k}}{\partial a_{j}}
$$

For edge-element based $\mathrm{FE}$, for instance, the entity $K_{i j}^{\prime}$ is computed from 


$$
K_{i j}^{\prime}=2 \sum_{k} \sum_{k^{\prime}} a_{k} a_{k^{\prime}} \int_{v} \frac{\partial v}{\partial B^{2}}\left(\operatorname{curl} \boldsymbol{N}_{i} \cdot \operatorname{curl} \boldsymbol{N}_{k}\right)\left(\operatorname{curl} \boldsymbol{N}_{j} \cdot \operatorname{curl} \boldsymbol{N}_{k^{\prime}}\right) d v .
$$

By solving the adjoint equation

$$
\left([K]+\left[K^{\prime}\right]\right) \overline{\boldsymbol{a}}=\frac{\partial f}{\partial \boldsymbol{a}},
$$

the sensitivity and the standard deviation can be computed from (4) to (6). Note that, the unknown vector $\boldsymbol{a}$ and permeability at each finite element in $[K]$ and $\left[K^{\prime}\right]$ must be obtained by solving (1) based on its linearization with e.g., the Newton-Raphson method.

\section{ROBUST OPTIMIZATION BASED ON AVM}

As mentioned in the first section, the present method evaluates the sensitivities in objective and/or constraint functions to consider their deviations. Here, let us consider how to impose the robustness for the objective function $f$. To do so, the original function $f$ is modified as

$$
f \rightarrow f \mp \alpha \sigma_{f},
$$

where - and + correspond to maximizing and minimizing problems, respectively. Moreover, $\alpha$ is a given constant which expresses the degree of the deviation. As $\alpha$ is increased, the optimal solution is expected to become more robust. The standard deviation $\sigma_{f}$ is computed from (5) or (6).

We next consider modification of constraints, say, $g<0$. We replace this condition with

$$
g+\alpha \sigma_{g}<0 .
$$

For $g>0$, we use a similar replacement with $g-\alpha \sigma_{g}>0$.

The present method would require nearly double computational cost in comparison with that of usual nonrobust optimizations because the adjoint equation must be solved in addition to system equation in an optimization process. However, this computational cost is considerably lower than that of the conventional robust optimization method which makes sampling around candidate solutions for robustness evaluation. One of the merits of the present method is in its simplicity. Due to this merit, it could be applied to a wide range of optimization methods.

\section{NUMERICAL RESULTS}

To test the validity of the present method, it is applied to two numerical examples. In the optimization, the realcoded clonal selection algorithm (RCSA) [Campelo et al., 2005] is employed as the optimization method. In our experiences, RCSA has similar performances in comparison with other stochastic methods such as genetic algorithm and evolutionary algorithm. The procedure of RCSA is as follows:

(a) generate $n_{\text {pop }}$ individuals,

(b) evaluate objective function and constraints for each individual,

(c) if convergence criterion is fulfilled, then go to (i),

(d) eliminate worst $P \%$ of individuals,

(e) generate certain number of clones regarding performance of individuals,

(f) execute mutation for each clone and selection of the fittest,

(g) generate random individuals and add them to population to keep $n_{p o p}$ unchanged,

(h) return to (b),

(i) end.

\section{A. Superconducting Magnetic Energy Storage}

TEAM workshop problem \#22, superconducting magnetic energy storage (SMES), shown in Fig.1 [Alotto, et al., 1998], is chosen as the first problem. Because this model has the axial symmetry to z-axis, AVM and FEM are formulated in the cylindrical coordinate system, and 2-D FEM is employed. The analysis domain is $20 \times 20$ $[\mathrm{m}]$. 


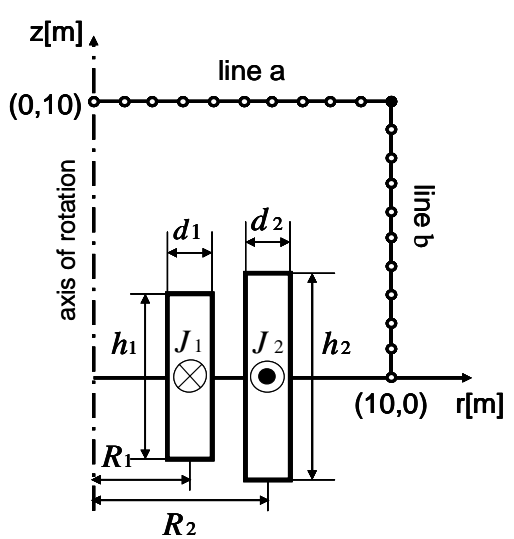

Fig.1 SMES model

In this example, the optimization problem is defined by

$$
\text { minimize : } f=\frac{\left|B_{\text {stray }}\right|^{2}}{\left|B_{\text {norm }}\right|^{2}}+\frac{\mid \text { Energy }-E_{\text {ref }} \mid}{E_{\text {ref }}},
$$

where $B_{\text {norm }}$ and $E_{\text {ref }}$ are set to $3.0[\mathrm{mT}]$ and $180[\mathrm{MJ}]$, respectively. $B_{\text {stray }}$ is evaluated as follows:

$$
B_{\text {stray }}=\sqrt{\frac{\sum_{i=1}^{21}\left|B_{\text {stray }, i}\right|^{2}}{21}},
$$

where $B_{\text {stray }, i}$ is the magnetic induction evaluated at 21 points on line a and b. In addition, the constraint condition is defined as

$$
-6.4|\boldsymbol{B}|+54.0 \geq|\boldsymbol{J}|,
$$

where $|\boldsymbol{B}|$ and $|\boldsymbol{J}|$ are the maximum induction and the current density in each coil, respectively. Inequality (16) denotes the quench condition which must be fulfilled to keep superconducting state.

To solve (14), design parameters $R_{2}, h_{2}, d_{2}, J_{1}$ and $J_{2}$ are chosen as the variables. Here, the standard deviations in $R_{2}, h_{2}$, and $d_{2}$ are considered, which are set to $1 \%$ of those values. On the other hand, $R_{1}, h_{1}$ and $d_{1}$ are fixed at 2.0, 1.6 and $0.27[\mathrm{~m}]$, respectively.

The value of $\alpha$ in (12) is set to 1.0. $n_{\text {pop }}$ and $P$ in RCSA are set to 50 and $50 \%$, respectively. In the mutation, random noise of deviation $5.0 \times 10^{-3}$ is added to the normalized shaped parameters.

The resultant values of parameters are summarized in TABLE I. The performances of obtained solutions are shown in TABLE II. It can be seen that both optimal solutions have almost the same performances, and we can confirm that the solution obtained by the present method is a bit superior to that obtained by the conventional non-robust optimization method from the view point of $B_{\text {stray }}$. In TABLE III, the values of objective function $f$ and its standard deviation are summarized. Cleary, the solution obtained by the present method is more insensitive than that obtained by the non-robust optimization. Here, let us consider $\left(f+\sigma_{f}\right)$ to understand the performances of obtained solutions under the effects of deviations in parameters. We can find that the solution obtained by the present method works better than the non-robust solution when the parameters have some deviations. Finally, the required computational time are shown in TABLE IV. The present method requires almost double computational cost as that of the non-robust optimization. It would be enough reasonable because we required 16690 [sec] to obtain a robust solution by using a robust optimization method introduced in [Steiner et al., 2004].

From these results it is concluded that the present method works well to obtain robust optimal solutions, and that it could be used for practical purposes from view point of computational time.

\begin{tabular}{|c|c|c|}
\hline & non-robust & present \\
\hline$\overline{\overline{R_{2}[\mathrm{~m}]}}$ & 2.80 & 2.83 \\
\hline
\end{tabular}

TABLE I

Resultant parameters of SMES 


\begin{tabular}{ccc}
$h_{2}[\mathrm{~m}]$ & 2.12 & 3.82 \\
$d_{2}[\mathrm{~m}]$ & 0.147 & 0.102 \\
$J_{1}\left[\mathrm{~A} / \mathrm{mm}^{2}\right]$ & 25.8 & 25.4 \\
$J_{2}\left[\mathrm{~A} / \mathrm{mm}^{2}\right]$ & -18.5 & -14.2 \\
\hline
\end{tabular}

TABLE II

Performances of obtained solutions

\begin{tabular}{ccc}
\hline & non-robust & present \\
\hline \hline$B_{\text {stray }}[\mathrm{T}]$ & $5.50 \times 10^{-4}$ & $7.52 \times 10^{-5}$ \\
Energy & 180 & 180 \\
{$[\mathrm{MJ}]$} & & \\
\hline
\end{tabular}

TABLE III

Resultant values of $f$ and standard deviation (SMES)

\begin{tabular}{ccc}
\hline & non-robust & present \\
\hline \hline$f$ & $3.66 \times 10^{-2}$ & $1.41 \times 10^{-3}$ \\
$\sigma_{f}$ & $8.60 \times 10^{-2}$ & $1.77 \times 10^{-2}$ \\
\hline$f+\sigma_{f}$ & $1.23 \times 10^{-1}$ & $1.91 \times 10^{-2}$ \\
\hline
\end{tabular}

TABLE IV

Required CPU time. (C-shaped core)

\begin{tabular}{ccc}
\hline & non-robust & present \\
\hline \hline $\begin{array}{c}\text { CPU time } \\
\text { [sec] }\end{array}$ & 3153 & 5556 \\
\hline \multicolumn{2}{c}{ CPU: Xeon5160(3GHz), memory: 8GB. }
\end{tabular}

\section{B. C-shaped core}

We consider the C-shaped magnet shown in Fig.2. In this model, magnetic field around a slice of C-shaped magnet is computed by 3-D FEM. The analysis domain is $700 \times 300 \times 10$ [mm], and is subdivided into 135,300 tetrahedral elements. The number of unknowns is 204,849 .

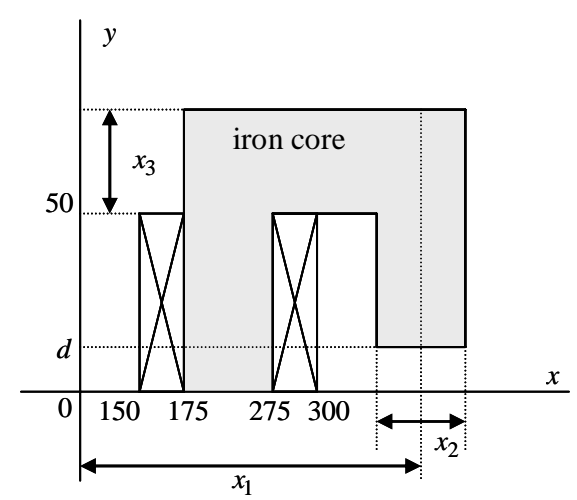

Fig.2 C-shaped core (upper half). Unit in [mm].

The permeability of iron core is assumed to be non-linear and the magnetostatic equation is solved by ICCG method coupled with Newton-Raphson iterations. The B-H characteristic in the iron core is shown in Fig.3. 


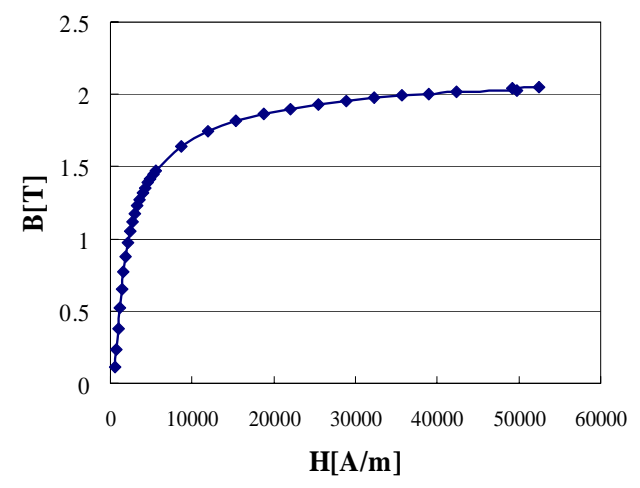

Fig.3 B-H characteristic.

In this example, the optimization problem is defined by

$$
\text { minimize }: f=\sum_{i=1}^{3}\left(B_{y i}-B_{r e f}\right)^{2},
$$

where $B_{y i}$, for $i=1, \ldots, 3$, denotes $y$-component of magnetic induction $\boldsymbol{B}$ at observation points $x=400,440$ and $480[\mathrm{~mm}]$ on $x$-axis. It is assumed that the design parameters $x_{i}$, for $i=1, \ldots, 3$, are linearly independent of each other, and those standard deviation is assumed to be $0.01 x_{i}$. The coil current and $B_{\text {ref }}$ are set to 5000[AT] and $0.8[\mathrm{~T}]$, respectively. The value of $\alpha$ in (12) is set to $1.0 . n_{\text {pop }}$ and $P$ in RCSA are set to 20 and 50\%, respectively. In the mutation, random noise of deviation $5.0 \times 10^{-3}$ is added to the normalized shaped parameters.

The resultant values of design parameters are shown in TABLE V. TABLE VI summarizes the values of magnetic induction at each observation point for the conventional non-robust and present robust optimizations, where $f$ and $f+\sigma_{f}$ are minimized respectively. It can be seen that there are no differences in the resultant values of magnetic induction. Moreover, TABLE VII shows the resultant values of $f$ and its standard deviation. It can be seen that the standard deviation in $f$ obtained by the present method is much smaller than that obtained by the non-robust optimization. It can be found that the magnetic induction corresponding to the optimal solution obtained by the non-robust optimization would vary $70[\mathrm{mT}]$ in average from the optimal values due to the parameter deviation. On the other hand, since the standard deviation for the present method is $3.9 \times 10^{-6}$, the corresponding deviation in the magnetic induction is around $2.3[\mu \mathrm{T}]$. It is concluded that the present method provides the robust optimal solution in comparison with the conventional non-robust optimization. Finally, the required computational time is summarized in TABLE VIII. The present method requires almost double computational cost as that of the non-robust optimization. As mentioned in the first section, the computational cost of the conventional robust optimization which carries out sampling at grid points is $2^{n}$ times as much as the non-robust optimization. Because $n=3$ in this example, the present method is more effective than the conventional robust optimization method.

TABLE V

Resultant values of design parameters.

\begin{tabular}{cccc}
\hline & $x_{1}$ & $x_{2}$ & $x_{3}$ \\
\hline \hline non-robust & 443 & 105 & 78.3 \\
present & 452 & 123 & 94.7 \\
\hline
\end{tabular}

Unit in [mm].

TABLE VI

Magnetic induction at observation points.

\begin{tabular}{cccc}
\hline & $B_{y 1}$ & $B_{y 2}$ & $B_{y 3}$ \\
\hline \hline non-robust & 800 & 802 & 798 \\
present & 800 & 802 & 798 \\
\hline
\end{tabular}

Unit in [mT].

TABLE VII

Resultant values of $f$ and standard deviation. 


\begin{tabular}{|c|c|c|}
\hline & $f$ & $\sigma_{f}$ \\
\hline $\begin{array}{l}\text { non-robust } \\
\text { present }\end{array}$ & $\begin{array}{l}8.78 \times 10^{-6} \\
7.50 \times 10^{-6}\end{array}$ & $\begin{array}{c}0.128 \\
3.90 \times 10^{-6}\end{array}$ \\
\hline \multicolumn{3}{|c|}{ Unit in $\left[\mathrm{T}^{2}\right]$} \\
\hline \multicolumn{3}{|c|}{$\begin{array}{c}\text { TABLE VIII } \\
\text { Required CPU time. (C-shaped magnet) }\end{array}$} \\
\hline & non-robust & present \\
\hline $\begin{array}{l}\text { CPU time } \\
\text { [min] }\end{array}$ & 2093 & 3888 \\
\hline
\end{tabular}

\section{CONCLUSIONS}

In this paper, a novel robust optimization method based on AVM has been introduced. The validity and availability are tested by applying the present method to 2-D and 3-D electromagnetic problems. The present method seeks for the robust optimal solutions which have small deviations in objective function. Moreover, it can find the robust solution within reasonable computational time. One of our future works is to consider a deviation in constraint conditions, e.g., quench condition for the SMES system. In general, when a solution violates constraint condition, the optimized system does not work well, or in the worst case the system falls into breakdown. Hence, in optimizations, the deviations in constraint condition should be treated more rigidly.

\section{REFERENCES}

Steiner, G., Weber, A., Magele, C. (2004) "Managing uncertainties in electromagnetic design problems with robust optimization,” IEEE Transactions on Magnetics, vol.40, no.2, pp. 1094-1097.

Maruyama, T., Igarashi, H. (2008) “An Effective Robust Optimization Based on Genetic Algorithm,” IEEE Transactions of Magnetics, vol.44, no.6, pp.990-993.

Akel, H., Webb, J.P., (2006) "Design sensitivities for scattering-matrix calculation with tetrahedral edge elements,” IEEE Transactions of Magnetics, vol.36, no 4, pp.1043-1046.

Okamoto, Y., Akiyama, K., Takahashi, N. (2006) "3-D topology optimization of single-pole-type head by using desigh sensitivity analysis,” IEEE Transactions of Magnetics, vol.42, no.4, pp.1087-1091.

Sikora, R., Kaminska, A., Sikora, J. (1996) "The inverse problem solution for infinite regions using the impedance tomography techniques,” IEEE Transactions of Magnetics, vol.32, no.3, pp.1294-1297.

Campelo, F., Guimaraes, F. G., Igarashi, H., Ramirez, J. A. (2005) "A Clonal Selection Algorithm for Optimizaiton in Electromagnetics,” IEEE Transactions of Magnetics, vol.41, no.5, pp.1736-1739.

Alotto, P. G., Eranda, C., Brandstatter, B., Furntratt, G. Magele, C., Moli, G. (1998) "Stochastic algorithms in electromagnetic optimization,” IEEE Transactions of Magnetics, vol.34, no.5, pp.3674-3684. 
Takayuki Maruyama, Kota Watanabe and Hajime Igarashi

Graduation School of Information Science and Technology, Hokkaido University,

E-mail: maruyama@em-si.eng.hokudai.ac.jp, watanabe@ssi.ist.hokudai.ac.jp, igarashi@ssi.ist.hokudai.ac.jp

Kita 14, Nishi 9, Kita-ku, Sapporo, Hokkaido, 060-0814, Japan

\section{T. Maruyama}

He is currently a doctoral course student of graduate school of information science and technology of Hokkaido university.

K. Watanabe

He joined department of electric engineering of Hokkaido University in 1999, and is currently an assistant professor at graduate school of information science and technology of Hokkaido university.

H. Igarashi

He joined department of electric engineering of Hokkaido University in 1989, and is currently a professor at graduate school of information science and technology of Hokkaido university. He was a guest researcher at technical university of Berlin supported by Alexander von Humboldt foundation from 1995 to 1998. 